Running head: ESTIMATING COSTS OF PREVENTIVE INTERVENTIONS

\title{
Estimating the Costs of Preventive Interventions
}

E. Michael Foster, Ph.D.

School of Public Health

University of North Carolina

Rosenau Hall, Campus Box\# 7445

Chapel Hill, NC 27599-7445

emfoster@unc.edu

919.966.3773

Michele M. Porter, M.S

Tim S. Ayers, Ph.D.

Debra Kaplan, Ph.D.

Irwin Sandler, Ph.D.

The Prevention Research Center

Arizona State University

Psychology-North

900 S. McAllister

Tempe, AZ 85287-6005

480 965-7420 - Office

480 965-5430 Fax

Please direct corresponding to the first author.

Support for this research was provided by the National Institute of Mental Health grant 2RO1MH49155.

Key words: Cost estimates, Prevention, Family Bereavement Program, Evaluation 


\begin{abstract}
The goal of this article is to improve the practice and reporting of cost estimates of prevention programs. It reviews the steps in estimating the costs of an intervention and the principles that should guide estimation. We then review prior efforts to estimate intervention costs using a sample of well-known but diverse studies. Finally, we illustrate the principles with an example, the Family Bereavement Program. We conclude that example by discussing whether and how the costs of the intervention might differ when implemented in a real-world setting.
\end{abstract}


Interest in the economic evaluation of prevention programs is growing rapidly.

Regardless of whether these analyses involve cost-effectiveness, benefit-cost analyses or analysis of public expenditures, the foundation for this research is a measure of the costs of the intervention evaluated. Cost estimates have other important uses. Policy makers, for example, need this information in order to plan implementations and budget appropriately. As discussed below, this issue seems especially important as many interventions are moving out of the realm of efficacy studies and into real-world settings. Estimates of intervention cost inevitably require some modification as the program is transported into community agencies; different inputs may be used or different prices may be paid for the same input. For that reason, a good understanding not only of the bottom line but also of the inputs and their value is required for applying estimates from efficacy studies to real-world contexts.

For these and other reasons, a sound cost estimate is essential to any economic analysisor indeed, any evaluation-of a prevention trial. At first glance, this task may seem to involve only a straightforward application of the principles of sound bookkeeping. However, as discussed below, estimating the costs of an intervention raises difficult conceptual issues. In some cases, those issues have no ideal or unique solution. In many cases, the answer is to "try it several ways”. Such an approach seems logical, but if the resulting estimates will be compared across studies, then at least one of the several ways have to be common across studies. Furthermore, the procedures used and assumptions made have to be stated explicitly so other researchers can understand how the estimates were generated.

More generally, as the economic evaluation of prevention grows, the field likely will benefit from a general framework for estimating program costs. Such a framework exists in health services research (Gold et al. 1996). That work represents a good starting point, but 
programs for the prevention of behavioral or substance abuse problems often take the researcher beyond health care facilities and into schools and other agencies or facilities. Working in these settings raises a series of new and challenging conceptual issues.

The goal of this article is to improve the practice and reporting of cost estimates of prevention programs. It reviews the steps in estimating the costs of an intervention and the principles that should guide estimation. We then review prior efforts to estimate intervention costs using a sample of well-known but disparate studies. Finally, we illustrate the principles with an example, the Family Bereavement Program. We estimate those costs from the perspective of a funding agency as well as from a social perspective, which includes the costs of participation for intervention families. We conclude that example by discussing whether and how the costs of the intervention might differ when implemented in a real-world setting. 


\section{Prior Research}

Prior research in health economics provides guidance on how to measure the costs of an intervention. Prior research is generally consistent with those principles, but the specific methodologies used often vary somewhat. As a result, the resulting estimates are difficult to compare.

Measuring the costs of an intervention.

An extensive literature in economics enumerates the steps in estimating the costs of a social program in varying ways, but the different approaches generally include six key tasks (e.g., Thompson 1980). These tasks involve

(1) clearly stating the intervention or activity for which the costs are estimated;

(2) establishing the perspective and scope of the study;

(3) identifying the program inputs;

(4) measuring their use;

(5) valuing those inputs and

(6) conducting sensitivity analyses.

This subsection describes these steps and identifies key economic principles that shape how each should be conducted.

Step 1: Clearly stating the activity or program for which costs are being estimated. This issue is critical and more subtle than it might seem. For example, a prominent issue in prevention science involves moving efficacious interventions from research settings into the community. Policy makers and even researchers may be more interested in the costs of the latter 
than the former. However, the first step in estimating costs involves the costs of the intervention as delivered, which initially is likely to be in research settings. Those costs and the resources to which they correspond were used to generate observed outcomes. As with outcomes, anything else is somewhat speculative. Admittedly, such speculation can be informed and useful, but the sound estimation of the costs of the program as delivered should be reported and lays the foundation for any adjustments that follow. Those adjustments may involve different inputs or different payments, but they are in fact adjustments that build on the costs as actually observed.

Step 2: Establish the perspective and scope of the study. The second step is to establish the study's perspective and, relatedly, the scope of the study. The former refers to the perspective from which costs are assessed. Common perspectives include that of participants, the funding agency, or society as a whole. The last generally includes all others and is of primary interest to economists. The societal perspective permits one to fully assess the costs of the intervention relative to other possible uses of the funds, including non-health care. Only from a social perspective can we fully capture the influence of the program on other systems and parties (Gold et al. 1996).

However, alternative perspectives are often useful for other purposes. Costs estimated from the agency perspective can facilitate public budgeting and identifying the distribution of a program's burden across agencies. The latter might be used to draw funding from different sources as the program evolves or is expanded. Furthermore, the perspective of participating families may be especially relevant for understanding their willingness to participate initially or to remain in the program over time.

Some costs may matter for some perspectives but not others. For example, participation 
may create costs for intervention families. Only to the extent that families are reimbursed would these costs appear on program budgets. In that case, the preponderance of these costs would not count from the perspective of the funder.

In some cases, whether an intervention creates costs or benefits depends on ones’ perspective. For example, a hypothetical job training program may reduce welfare payments to participants. The reduction in those payments is a cost for the poor women in the program. Those savings, however, are a benefit for taxpayers. From a societal perspective, therefore, the two largely cancel each other. (The net benefits for society involve the reduced costs of administering the transfer payments. Unless the scope of the program is fairly large, those costs will remain unchanged.)

Relatedly, the study must specify a scope, which involves the breadth or type of costs or resources captured. To some extent, the researcher must draw a boundary between the resources that are and are not included in the cost analysis. The effects of an intervention on resource use ripple outwards almost endlessly, and the costs of measuring every conceivable effect would be exorbitant.

Scope can be defined in terms of the four types of costs one encounters in health care: medical, non-medical, productivity and intangible costs (Haddix, Teutsch, and Corso 2003). For a physical health service, non-medical costs might involve the costs of transportation. In the case of mental health, these non-medical costs may be especially important and involve a multitude of child-serving sectors, such as schools, juvenile justice and child welfare. Productivity costs represent time costs; they correspond to reductions in time on the job or in housework. They can involve the provision of treatment or may stem from the illness itself. 
Intangible costs involve non-monetary costs that are difficult or impossible to measure, such as stigma. The scope of a study almost always includes the medical and related non-medical costs of services; studies differ in whether these broader costs are included.

An added dimension of scope is temporal—it involves the time frame and time period for the study (Haddix, Teutsch, and Corso 2003). The latter refers to the period during which the study is being conducted. That period often can be segmented into phases, such as startup or developmental and intervention delivery. The former often involve fixed or one-time costs incurred as the project is designed and refined. One also may present costs during sub-periods of the delivery period. These intervals may involve calendar years and/or intervention cohorts. Such information might be important in understanding how the costs of the intervention rise as the project matures or its scale expands or how costs decline as the staff become more expert and need less training and supervision.

The time frame over which costs are measured can extend beyond even the life of the intervention itself or even the time period for the study itself (i.e., the period during which data are collected). Suppose, for example, an intervention links families to services. They may continue to use those services after the intervention ends. Unless the perspective of the study is very limited, those services should be included as part of the program's costs. Costs that occur after the study period but during the time frame generally require additional data from other sources. For example, an intervention to reduce behavior problems may improve the chance that participants attend college. Clearly such attendance has many benefits that one would want to capture but the related educational expects represent costs of the intervention as well.

To some extent, specifying the study's perspective does delimit the costs included. As 
noted, if one's perspective is that of the funding agency, then participant time may be excluded, at least beyond any payments made for participation. A societal perspective inevitably involves a broader scope. One means for circumscribing the scope involves the likelihood and magnitude of the effects involved. The latter involves both the amount of resources involved as well as their value. Another consideration involves the perspective and scope shaping the analyses of program benefits. Consider, for example, of an early childhood education program (such as Perry Preschool, discussed below). If one intends to include greater college attendance (and the resulting increase in earnings) as part of the program's benefits, then one should include the costs of college as part of the costs of the intervention.

One basis for restricting the scope of the analysis is not useful-whether the costs were intentional or anticipated as part of the intervention's design. This distinction is irrelevant; what matters is that the resources were used and not available for other uses. For example, participants in a parenting intervention may use more mental health services because they become comfortable dealing with mental health professionals. The resulting expenditures are part of the intervention's costs, even if the effect is unintended or unforeseen.

As discussed above, study resources are scarce, and so the scope and perspective of a given study may be more limited than is ideal. However, clearly stating the scope and perspective is essential, especially if one is interested in comparing the costs of interventions across studies. If two studies differ in scope and perspective, determining which intervention is more costly is difficult or impossible. For example, two interventions may differ in participant time required; ignoring those costs artificially makes the more intensive program look more favorable. If that difference is stated, however, one can at least partially adjust comparisons for that difference or at least interpret differences in included costs in light of those that were 
omitted.

Step 3: Identify the program inputs. The second step in estimating costs involves identifying program inputs. The resulting list should be comprehensive and consistent with the scope and perspective of the study. This list should include inputs even if measuring their use of resources or valuing them in dollar terms is difficult (Gold et al. 1996).

A key principle in measuring those inputs involves measuring the marginal effect of the activity or intervention of interest. That effect corresponds to the incremental effect of the program. That effect depends on a variety of factors, such as the study's time frame. If the time frame is short, the marginal (opportunity) cost of treating an additional child is possibly quite low and does not include the costs of training intervention personnel. However, if the study perspective is longer term, then one must recognize that intervention personnel would require retraining or that turnover would require that new personnel be hired. In that case, the long-term marginal costs must include an appropriate portion of the costs of training personnel. ${ }^{1}$

Step 4: Measure the use of those inputs. The fourth step involves measuring the use of those inputs. Project budgets often serve as the key input for this task, and to the degree that the costs involve only tangible resources under the control of the evaluation, those data should be sufficient. However, in many instances-especially when the perspective is societal-researchers must move beyond project budgets. In the case of participant time, this step can involve collecting additional data. One might ask participants to track their time traveling to and from intervention activities or preparing for training sessions. Asking participants to provide such information may be infeasible. For example, the cost estimation may be retrospective, and the

\footnotetext{
${ }^{1}$ In essence, training of personnel would be treated as “equipment” and would be depreciated over time. For details, see Foster, Shelton-Johnson and Taylor, 2004.
} 
study may have no further contact with participants. In that case, an alternative is to develop a reasonable estimate based on other studies (combined with information regarding the intervention of interest).

A key issue that arises at this point-especially in efficacy studies-involves dividing shared inputs between intervention delivery and research. In the case of personnel, one can divide these inputs using time sampling or time diaries (Hargreaves et al. 1998). In the case of space, that space can be divided in terms of size and use and/or based on the time allocation of the persons involved. ${ }^{2}$

Step 5: Value the resources in dollar terms. The fifth step involves valuing the resources involved in dollar terms. How this step is accomplished depends on the study perspective. Fortunately, in many instances, project budgets provide the necessary information. However, even when payment data are available, they may not pertain to the perspective of interest and so require further adjustment. These adjustments are especially likely when the perspective of interest is societal. This issue arises commonly in health care, where prices are determined in non-competitive markets. The resulting prices may reflect a variety of considerations, such as the degree of competition among health care plans and/or facilities in the local market. In some cases, the necessary adjustment can be calculated using economic theory. (The theory of monopolistic markets and the elasticity of demand allow one to calculate the extent to which prices exceed the marginal cost of producing the good or service (Browning and Browning 1992)). In some instances, one can use alternative prices. For example, in costing out medications, one might use the wholesale rather than the retail price.

\footnotetext{
2 A related issue may arise in effectiveness studies involving service delivery in community agencies delivering multiple programs or services.
} 
These issues arise even outside of hospitals and medical offices. The value of university space is determined by a process that may have little to do with competitive markets. Payments made for this space may reflect a variety of considerations, and payments for similar space may vary widely. One alternative is to measure the costs of that space using rental rates for similar commercial property.

Further complexities arise in measuring the value of inputs provided in schools or other settings. In many cases, the facilities involved cannot or will not provide the necessary budgetary information. Furthermore, making sense of information provided may require more resources than the evaluation can provide. For example, few studies have the resources necessary to obtain budgetary data on the costs of special education in a local school. One alternative is to employ a "gross costing” strategy that relies on other estimates (Gold et al. 1996). Unfortunately, these estimates may be unavailable or may have serious limitations. Many of the systems involved vary substantially across communities, and cost estimates for special education developed in one community may have limited external validity.

Issues inherent to the notion of “opportunity costs” complicate matters still further. By their very definition, these costs involve opportunities foregone: "What would these resources been used for had they not been used for our project?” This answer to this question is inherently hypothetical. In some instances, the answer is fairly straightforward, especially where a market for the input exists. For example, one can imagine intervention personnel finding a job as a counselor or therapist, and their current wage is presumably a good proxy for what they could earn in those positions. This task becomes more complicated for inputs that have not been purchased recently or for which payments are not made, such as university space. 
Participant time in particular represents an input for which opportunity costs are difficult to value. Economists approach this problem by trying to link the value of time to the wages individuals earn working. The link is obvious for individuals who are paid hourly and who miss work to attend the program. However, what non-economists may find surprising is that economists use the wage rate to value leisure time. This practice reflects their understanding of the so-called labor-leisure tradeoff-in this framework, individuals could work well beyond 40 hours per week at their going wage (Borjas 2005; Ehrenberg and Smith 2006). From that perspective, that individuals choose not to work beyond the amount they are currently working means that the value of their leisure time actually exceeds their wage rate. (For further discussion, Foster, Shelton-Johnson and Taylor, 2004 or (Posnett and Jan 1996).)

Finally, note that resources should not be double-counted. For example, incentive payments and time costs should not both be counted. Like other inputs, parental time is valuable because it could have been used for other purposes. One opportunity foregone involves caring for ones children. To the extent the parents are reimbursed, they are compensated for foregone opportunities, and the costs to them are eliminated.

Step 6: Conduct sensitivity analyses. The final step in cost estimation involves examining the uncertainty related to key assumptions in calculating costs. As is apparent above, the analyst inevitably will encounter situations where he or she must pick between two imperfect alternatives. For example, in valuing persons' time, does one use their own wage rate or that of members of society on average? If one uses their own wage rate, then one values men and women's time differently, and a prostate cancer screening looks more expensive than breast cancer screening. One can reasonably argue for either alternative. The solution is to examine the sensitivity of study findings to the different alternatives (Gold et al. 1996). 


\section{Prior Research Providing Estimates of Intervention Costs}

To assess current practice in the field of prevention and intervention research, we examined five high-quality programs for which cost estimates are available and for which economic evaluations have been conducted. Three of these studies involve intensive centerbased child care: Chicago Parent-Child Centers (Reynolds et al. 2002), Abecederian (Maase and Barnett 2003), and Perry Preschool (Barnett 1996). While these programs differ in key ways (e.g., the number of years in which services are provided), policy makers and researchers certainly have been interested in whether and how their costs differ. We also examined three other studies--Old's studies of Nursing Home Visits (Olds et al. 1993) and two universal prevention programs designed to prevent alcohol and drug use by middle-schoolers (Spoth, Guyll, and Day 2002).

These studies are high quality and generally conform to the principles outlined above. For example, Barnett (1996) clearly specifies multiple perspectives and presents estimates for each. Where only a single perspective is specified, the studies are quite clear as to the study perspective. Olds (1993), for example, clearly states that his analyses are limited to the public perspective.

The studies also attempt to estimate marginal costs. For example, the child care studies are sensitive to the fact that children in the control group also received services and adjust their analyses for the costs of those services — these studies estimate the costs of the program over and above the costs of services children otherwise would have received. The studies also describe the costs of the program as actually implemented. Maase and Barnett (2003) estimate the costs of the Abcederian project as delivered but also estimate the costs were the program to be 
delivered in two real world settings (public schools and day care).

The studies also make sensible choices in terms of balancing accuracy against study resources. For example, Maase and Barnett (2003) do not attempt to estimate the costs of special education services actually delivered to study children. Rather, they rely on external estimates of the costs of those services. The studies also include sensitivity analyses involving a reasonable range of key assumptions.

In spite of the studies' strengths, however, the bottom line of our review is that comparing cost estimates across these studies is difficult. Our inability to do so largely reflect the limited detail reported. For the most part, Barnett (1996) provides sufficient detail, but that study comprises an entire book. However, even in the space of a single article, the authors differ in how much detail is provided. Maase and Barnett (2003) provide the least detail, perhaps feeling that the detail is available in an unpublished dissertation.

Available detail does call into question whether the estimates in these studies can be compared. For example, the studies differ a good bit in whether and how administrative and other overhead costs are included. Two studies include the costs of training intervention personnel (Olds and Spoth), but the others do not. The studies also differ in whether and how participant time costs are included. Olds does not include these costs, which is consistent with the study's perspective. The failure to provide the information, however, still represents a barrier to comparing that intervention's costs with that of other programs. Spoth and colleagues (2002) ignore time costs, even though the study's perspective is societal. Barnett (1996) excludes time costs associated with home visits, arguing that offset by the benefits of the intervention. Whether this claim is correct is difficult to ascertain. Parents' decisions to participate reflect 
anticipated future benefits as well as the direct utility provided by the intervention (i.e., whether the home visits are enjoyable). Reynolds and colleagues (2002) do include opportunity costs for parental participation, but how these were calculated is unclear (Reynolds et al. 2002).

This situation is unfortunate. Several recent reports compare these studies in terms of the magnitude of their benefits (Aos et al. 2004; Karoly et al. 2001). A necessary condition for such comparisons, however, is that the cost estimates be comparable. Given the lack of such comparability, cross-study comparisons of cost-effectiveness are ambiguous or meaningless. 


\section{An Illustration: The Family Bereavement Program}

The Family Bereavement Program (FBP) is a psycho-educational preventive intervention program targeted at children, adolescents and the caregivers of recently bereaved children. The intervention includes twelve weekly, two-hour group sessions and two individual sessions; all occur within a three month period. Separate group programs have been developed for caregivers, children and adolescents.

The FBP rests on a small theory approach (Lipsey, 1990) to target factors and resources that might prevent bereaved children's mental health problems. Targeted mediators were selected based on empirical evidence of a relation between these variables and mental health problems during childhood or adulthood of those who had been parentally bereaved as children (Ayers et al., 2004). In particular, the caregiver program promotes the quality of the caregiver/child relationship and the mental health of the caregiver; improves discipline practices and reduces the children's exposure to stressful negative events. Both the child and adolescent group programs targeted the same mediators but in developmentally appropriate ways. The targeted mediators of these programs were to promote a positive caregiver/child relationship and increase children's coping, coping efficacy, self-esteem and appropriate control related beliefs, while decreasing negative appraisals and their inhibition of feeling expressions (Ayers et al., 2004).

The FBP intervention groups were co-led by two MA level clinicians or clinicians with comparable work experience. Group leaders received 40 hours of training prior to the start of the program, which consisted of readings and didactic presentations about the theoretical and empirical bases for the program, videotapes of prior program sessions, and role-plays of session material. In addition, group leaders received 2 hours of training each week to ensure they were 
sufficiently prepared on the content and process of the session prior to training on the session. Further, supervision following each session (1.5 hours) used video tapes of the session, addressed problems of implementation, and tracked ways to fit the program to the needs of each group member.

Sandler and colleagues (Sandler et al., 2003) conducted an experimental trial of the FBP intervention with a sample of 244 bereaved children (aged 8-16) and their families ( $\mathrm{N}=156)$ comparing the FBP group intervention to a guided reading (i.e. bibliotherapy) program. Families were recruited in six cohorts over the course of three years, and once enrolled in the study were randomly assigned to one of the two conditions. Strengths of the program evaluation included the use of a randomized controlled experimental design, inclusion of a large community-based, heterogeneous sample of participants, assessment of family and child resilience resources as well as mental health problems, use of multiple reporters and methods to assess outcomes, and inclusion of an 11-month follow-up to assess maintenance of program effects.

At immediate post-test, program participants had improved on a range of targeted mediators including caregiver-child relationship, caregiver mental health, use of active coping strategies, and (reduced) inhibition of feeling expression (Sandler et al., 2003). At the 11-month follow-up the program participants showed continued improvement on many of these targeted mediators such as sharing of feelings and a behavioral observational rating of generating problem solutions. Additionally, families in which parent and child reported poor parenting practices at the start of the program showed greater improvement in these behaviors at post-test relative to the families in the guided reading program. Few differences in symptomatology between conditions existed at immediate post-test. However, at 11-month follow-up, caregivers reported fewer internalizing problems for children who were higher on those problems at 
baseline. At 11-month follow-up, girls involved in the program had lower internalizing and externalizing problems according to parent and child reports.

\section{Methods: Budgetary Costs of FBP}

The first perspective for our analyses of FBP costs is that of a public agency or other funding source. For that reason, our analyses focus on explicit costs, including personnel, consultants, benefits, intervention direct expenses, travel, miscellaneous supplies, and equipment. However, we also included implicit costs - i.e., resources for which no explicit payments were made. These costs included intervention space, volunteered time by faculty and clinical staff and associated fringe benefits. Typically, these costs are included only when examining costs from a societal perspective. We include them here, however, because they inform our efforts below to estimate the costs of the intervention when implemented in community settings. These costs include that of space as well as project administration provided by study researchers (who provided more time than was actually funded under the grant).

To calculate intervention costs, comprehensive budget spread sheets from June 1996 through September 2000 were examined. These budgets enumerated project inputs and generally provided data on related payments. A principal task was to divide expenses between research and intervention and to remove the former from our tabulations. Explicit expenses included faculty, field managers, research associates, graduate students, interviewers, group leaders, hourly employees, administrative staff, and benefits. Fringe benefits (paid vacations, holidays, retirement, flexible spending plans) were included for all benefit-eligible employees, research-only expenses encompassed travel and subject fees among others. Printing, catering, counseling referral service, transportation and group snack and incentives were captured in the 
intervention-only category. Shared expenses, such as office supplies, were split between the intervention and research as described below. The costs of equipment purchased in the beginning of the project were spread across all six cohorts to accurately reflect costs (i.e., the depreciation of those resources over time).

We divided the study period into three intervals: development, piloting and intervention. Eighteen months from June 1996 through December 1997 were designated as development time, and two pilot studies occurred during this period. Between January 1998 and September 2000, the intervention was delivered to six cohorts. Beginning in January 1998, each year was divided in to an 'On Time' period or an 'Off Time' period. 'On Time' periods were September through May and were high in intervention activities. 'Off Time' periods were June through August when the intervention was not delivered and were low in intervention activities.

Budget spreadsheets were used to determine the amounts of resources used and payments made. The spreadsheets allowed us to distinguish expenses associated with development, pilots and delivery of the intervention. Each intervention period was linked to the associated cohort. All six cohorts had pre-intervention (recruitment, interviews, and training), intervention (intervention delivery) and post intervention (follow-up and debriefing) periods.

A key challenge in separating research from intervention costs involved personnel involved in both activities. These personnel included the program director, group leaders, and the study's field manager. To calculate personnel research ratios, detailed assessment of electronic diaries and time sheets for different personnel were examined. In particular, the FBP program director kept an electronic daily dairy of time spent on the Family Bereavement Program. Two 3-month time samples were selected to estimate the percentage of time devoted to 
intervention activities. The time samples were selected from both the On Time periods (Sept, Oct, and Nov) and the Off Time periods (June, July and August). A ratio of 55\% research to $45 \%$ intervention time was calculated for the On Time periods, and a ratio of $98 \%$ research to 2\% intervention was calculated for the Off Time periods. These time ratios were applied to other positions that engaged in similar activities to the program director's activities.

Group leaders delivered the weekly, 2-hour intervention. They were required to complete weekly timesheets that were broken down into nine separate categories of activities. The categories were group, training, supervision, co-leader practice, preparation for intervention, clinical notes, evaluation, telephone calls and interviews. The time sheets were used to allocate time spent on research (specifically, the "evaluation" category) and intervention activities across all nine categories. This information allowed us to allocate intervention time across the tasks involved, such as preparation and clinical notes versus actual contact with participants.

The field manager's intervention time was calculated based on the time sheets and on interviews with former field managers. Based on these sources, the field manager spent 38\% of his/her time in intervention-related activities. These activities largely involved participant recruitment. Since only $58 \%$ of the recruited sample was in the intervention, we included $22 \%$ (or 58\%*38\%) of his/her time as intervention costs. This ratio was applied to the field manager and field assistant's time across all six cohorts.

Some significant costs were implicit; these included the two senior investigators' time and associated benefits, volunteered clinical supervision, cost of intervention space and overhead. Both investigators spent more time on the project then what was reflected in their salaries. Based on time diaries, group supervision schedules and cohort analyses, costs were 
allocated for the unpaid time. Two cohorts had unpaid clinical supervision that was valued at the standard rate and applied as implicit costs to those cohorts. Space costs also were included. FBP was not charged for the use of rooms for intervention delivery, so we used the standard university rental rate to value this space. Project overhead was distributed on the same basis as the salaries of personnel with offices.

\section{Methods: Costs to Participants}

The costs to participants were of two types-transportation costs (time and mileage) and time related to actual intervention activities. Transportation costs were calculated using information on the location of intervention sessions and place of residence. Mileage and time spent traveling was determined using Mapquest.

The costs of participation also include time spent in intervention-related activities. Parents reported time spent in these activities each week on a brief instrument. Reported activities included Family Fun time as well as time spent practicing the skills they learned in the prior session.

Following economic theory, we valued participant time using self-reports on their occupation and information on their earnings using data from the Bureau of Labor Statistics. Earnings data were for individuals in Arizona and reflected 1990 Census data. 15 individuals were excluded from these calculations because they were not working.

Results: Budgetary Costs of FBP

Intervention costs were calculated at three levels, per family, per person, and per hour of contact, from two different perspectives, from that of the public agency (or payor) and society. With regard to the latter, the key omission is participant time costs. From a public agency 
perspective, which includes explicit costs (i.e., personnel, consultants, benefits, intervention direct expenses, travel, miscellaneous supplies, and equipment), the mean cost per family across cohorts was $\$ 4,424$ and ranges from $\$ 2,731$ to $\$ 6,957$. The mean cost per person was $\$ 1,624$ with a range from $\$ 940$ to $\$ 2,174$ across cohorts.

To determine the cost per hour of contact, we first assessed how many hours of contact each person in the intervention received. Caregivers and children each attended 12 2-hour group sessions and 2 1.5-hour individual sessions yielding a total of 27 hours. We also estimated that each person spent a half hour completing an initial intake interview. ${ }^{3}$ Thus, each person received 27.5 hours of contact in the intervention. The mean cost per hour of contact per person across cohorts was $\$ 59$, with a range from $\$ 34$ to $\$ 79$. Table 1 shows the explicit costs per family, per person and per hour of contact within each cohort.

-- Table 1 about here --

The costs per family, per person and per hour of contact from a societal perspective, including both explicit and implicit costs (i.e., space, volunteered time by faculty and clinical staff and associated fringe benefits) also were examined. The mean cost per family across cohorts was $\$ 5,006$, with a range from $\$ 3,354$ to $\$ 7,799$. The cost per person ranged from $\$ 1,155$ to $\$ 2,437$, with a mean of $\$ 1,836$. The mean cost per hour of contact per person across 6 cohorts was $\$ 67$, with a range from $\$ 42$ to $\$ 89$. Table 2 shows the explicit and implicit costs per family, per person and per hour of contact within each cohort.

-- Table 2 about here --

\footnotetext{
3 The actual interview lasted 90 minutes, but only 30 minutes was related to the intervention itself. The remainder was related to research.
} 
Where did the money go? (Direct contact v. administration)

A closer look at the breakdown of intervention costs pinpoints more specifically where the money was spent. Table 3 shows the breakdown of explicit costs within each cohort. The most expensive input for all cohorts is the group leader time followed by the training, supervision and faculty time. As one would expect, the bulk of costs involve group leaders preparing and delivering the intervention and their training and supervision.

-- Table 3 about here --

How did the costs vary across cohorts?

The total intervention costs varied across cohorts in that cohort 6 , with the most number of families $(\mathrm{n}=21)$, was the least expensive and cohort 4 , with the least number of families $(\mathrm{n}=$ 10), was the most expensive. Another interesting finding is that cohorts 2 and 3 each were comprised of 16 families yet the overall costs differed by $\$ 7,393$ (cohort 2: $\$ 67,655$; cohort 3: $\$ 60,262)$. This cost difference reflects a decrease in group leader time from cohort 2 to 3. Looking at cost per person between these two cohorts indicates that the cost is more expensive in cohort 3 than 2 (cohort 2: $\$ 1,611$; cohort 3: $\$ 1,629$ ) because cohort 2 is comprised of more family members compared to cohort 3 (cohort 2: $\mathrm{n}=42$; cohort 3 : $\mathrm{n}=37$ ).

What were the costs of development? (Pilot cohorts)

Other important costs include program development and two pilot interventions. Costs were assessed in terms of explicit and implicit costs. Table 4 shows the total costs and the costs per family for development and the pilot interventions. Somewhat surprisingly the costs of the pilot cohorts were no greater than those for subsequent cohorts. The table also reveals that the development costs were fairly substantial. Those costs were roughly equal to the costs of an 
additional cohort. The development costs encompassed the first six months of the grant prior to the pilots and this period was labor intensive due to preparation of the first version of the training and intervention materials.

\section{Results: Costs to Participants}

To simplify the task, we calculated travel distances and times for only two cohorts, one cohort for each of the two intervention sites. The calculations described above revealed that on average participants spent 40 minutes per week traveling back and forth to group meetings for a total of 8 hours over the 12 weeks. Travel involved 31 miles per week for a total of just over 368 miles. At a rate of 32\% per mile, mileage costs were estimated at $\$ 9.82$ per week or $\$ 118$.

In addition to travel time, individuals spent an additional 27 hours in intervention sessions (12 two-hour group meetings plus three hours of individual therapy). Participants reported spending 5.6 hours over the twelve weeks in Family Fun Time. Parents also reported spending 23 minutes per week practicing parenting skills for a total of 4.5 hours over the course of the program. Over 12 weeks, intervention-related time totaled 45.5 hours. Using the earnings information described above, this represents an average of $\$ 868$ per participant. (Average hourly wages or earnings were roughly \$19 per hour.) Taken together the transportation and participation costs total $\$ 986$.

-- Table 4 about here --

\section{Sensitivity Analyses}

A key input into these calculations involves the time distribution of the project director. We used this breakdown not only for his time but also for other key inputs. This figure was based on time sheets kept maintained during the actual project (rather than retrospective 
reports). However we only sampled those sheets from the beginning of the intervention. Those figures might have been lower for later periods due to more efficient use of time and resources. For example, not as much time may have been needed for preparation or training in later cohorts since the training materials had been developed and established in prior cohorts. This could decrease the intervention proportion of the costs by as much as 10 percent. This 10 percent decrease in the invention costs represents an overall reduction of just over $\$ 20,000$. On a per case basis, the effect is very small.

Another key feature of our calculations is the assumption that group leaders would have to be retrained for every cohort. Such training may not be required (especially in a real-world setting, as discussed below). If group leaders are trained once, the overall training costs would be reduced by $83 \%$. Instead of the current total cost of $\$ 12,057.00$ for training, it would only cost $\$ 2,010$ for the whole intervention or $\$ 335.00$ per cohort.

\section{The Costs of Delivering FBP in the Real World}

As the translation from efficacy to effectiveness trials and then to broader dissemination occurs, an inevitable question arises: how much will delivering the intervention cost in the "real world”? Two approaches are possible. One approach simply uses different per-unit costs based on the "standard rates" in the natural service delivery settings. This approach assumes that the quantity of inputs would remain the same. While somewhat informative, this approach ignores economic theory. If community agencies optimize their use of resources, then differences in input prices should create differences in how resources are used. For example, clinician salaries may be higher in community settings than those paid to researchers working in university settings. In that case, agencies should use less of the (relatively) more expensive input. This 
substitution of inputs should somewhat mute the effect of higher input prices.

A proper approach, therefore, would assume that the intervention would be modified in some form as it was moved into "natural service delivery settings (e.g. either increased or decreased clinical supervision, training etc) and to adjust overall costs based on costs associated with the efficacy or effectiveness trial. These two approaches can be combined to produce estimates that incorporate differences in both the prices and quantities of inputs used.

To understand how community agencies might modify the intervention, we contacted three agencies that specialized in the delivery of bereavement services in the Phoenix Metropolitan area. The agencies involved generally were outpatient mental health providers offering a range of outpatient services or specialty providers, focusing on bereavement services. Agency directors were interviewed about the delivery of a group psycho-education intervention such as the Family Bereavement Program. The agencies provided a broad range of bereavement services to families who had experienced the death of a loved one in a range of settings. Typically, masters-level staff delivered the intervention.

These interviews revealed key differences between service delivery in an efficacy trial and that in a community setting. First, the agencies differed in the time allocated for training, supervision, and preparation for the delivery of the intervention compared to the efficacy trial. Reflecting our discussion above, we reduced this cost to $1 / 6^{\text {th }}$ that (i.e., to once for all cohorts) reported in the efficacy study. This cost might actually be lower in community settings if training was conducted even less often.

Second, the agencies suggested that group leaders would be supervised quite differently. Respondents reported that most supervision would involve peer supervision. As a result, the 
ratio of supervision to contact would be much lower than in the efficacy trial. Based on the reports from these agencies on peer-to-peer supervision, the time in supervision was estimated to be only about one sixth of what was done during the efficacy trial.

Third, the agencies also reported that groups likely would not be staffed with two group leaders per group. The agencies typically use one leader, and as a result, co-leader practice would be unnecessary. Finally, the agency heads reported that the Group Facilitators would spend less time preparing to deliver the intervention. Based on the reported number of average hours Group Facilitators spent each week preparing to deliver the intervention the agency heads felt that they would only spend approximately a quarter of the time.

-- Table 5 about here --

Table 5 summarizes these differences and their implications for the distribution of facilitator time across activities. The two columns present the percentage breakdown for the efficacy study described above and a hypothetical "real world” setting. Taken together, these differences in service delivery across settings suggest that Group Facilitators would spend approximately 50\% of their time actually delivering the intervention. This figure is double that for the efficacy study. One can see that these changes imply a profound effect on the costs of the intervention. In essence, community settings would treat twice as many youth with the same personnel costs.

What would be a ballpark estimate of the overall costs of the intervention in the real world? Agency leaders also noted that they would not provide catered meals, transportation, babysitting and other ancillary services. However, all other costs that were associated with delivering the intervention were likely to be similar to those incurred during the efficacy trial. 
These costs include administrative expenses $(\$ 7,771)$, supplies $(\$ 6,303)$, equipment $(\$ 4,821)$ and costs associated with providing for the intervention space $(\$ 13,608)$. Calculation of overhead for office space needed to be adjusted since the calculation for the efficacy trial was based on total faculty and administrative costs * 33\%. Using this same approach with the reduced costs associated for faculty time, the total overhead for the intervention within a typical community setting would be estimated to be $\$ 9,394$. Including all of the adjusted costs associated with delivering the intervention in a natural service delivery setting (e.g. group facilitator, faculty and other costs) results in a total cost of $\$ 158,663$ for delivering the intervention to 90 families or 243 individuals. Thus, the cost of delivering the FBP intervention in a natural service delivery setting while making the modifications to the program outlined above would be $\$ 1,763$ per family, \$653 per person or \$24 per contact hour. This figure is less than half that for the efficacy trial (i.e. \$59 from a public agency perspective). 


\section{Discussion}

This paper outlines the principles by which the costs of an intervention should be estimated. The paper also illustrates those principles and corresponding procedures using information on a well-known intervention. The costs of the intervention examined are relatively modest, averaging about $\$ 4,000$ per family. These costs are especially reasonable when calculated on per-person and per-hour bases. The costs per hour (\$59) are quite comparable to the rates for outpatient therapy in many settings. For example, based on reviews of medical records for hundreds of children and youth in four communities, Foster and Jones (2004) find that total charges for outpatient mental health treatment averages \$81 per visit.

Our discussions with real-world providers suggest that the costs of providing these services will be substantially lower. Such services may even be less effective but still more costeffective if the reduction in costs is greater than the reduction in effectiveness. A full analysis of this question awaits effectiveness studies that will provide information on treatment impact when delivered in the real world. 
A key feature of these analyses is the inclusion of the costs to participating families. Those costs are substantial, totaling nearly $\$ 1,000$ dollars. Of course, participants reap many of the benefits of treatment, and so the intervention may prove quite cost-effective for them. However, these costs are sizable and may represent barriers to participation. For that reason, researchers and clinicians might bear these costs in mind when determining the number of sessions or the location of sessions. To date, the level of participation largely reflects an effort to maximize efficacy. It seems clear that those choices should reflect program costs as well. At this point, we know very little about the level of treatment (or dosage) and cost-effectiveness of interventions.

These estimates lay the groundwork for a full economic analysis. Because of its impact on the emotional and behavioral health of surviving family members, the potential economic consequences of bereavement are large. Some of these effects are difficult to measure (e.g., emotional trauma), but these effects are captured, at least to some extent, by changes in the use of health and mental health services by both children and adults. Costs of services for children's mental health problems for which bereaved children are at risk can be quite high. Illustratively, in the Fort Bragg study (Bickman et al. 1995), the mean costs for treatment of a case of child depression exceed $\$ 5000$ for 12 months and could range up to $\$ 40,000$. While direct evidence on the link between delinquency and bereavement is limited, Gregory (1965) found that bereaved children were more likely to be involved in the juvenile justice system, which creates large public and societal costs (Gregory 1965). Whether and how the benefits of the intervention offset its costs is a focus of ongoing research.

Second, the costs of the intervention varied a good bit across cohorts - this variation was driven by differences in cohort size. This finding need not imply that the number of families 
included in a cohort should be limitless in order to drive costs down. Costs are merely one factor to consider when determining cohort size. The current study is somewhat underpowered for examining variation in effectiveness across cohorts. Increases in group size may reduce average effectiveness but still be cost-effective. If true, such a finding would have important implications for how these services are delivered in community settings. In particular, it may be cheaper to have fewer agencies delivering to a larger catchment area than for multiple competing programs to offer the program. Furthermore, the agency would have to consider how often to offer such groups, balancing issues such as the timeliness of treatment with group size.

This issue speaks to broader considerations involving the design of interventions. In particular, many efficacy studies involve interventions that have been designed to maximize the likelihood of efficacy. However, the progress of the field from efficacy to effectiveness would be accelerated in the process were more seamless - in particular, if interventions initially were designed with an eye toward cost-effectiveness (Sandler et al., 2005). Such considerations include optimal (cost-effective) group size as well as the number of intervention components.

Our analyses are useful for understanding a single intervention targeted at a highly vulnerable group, but our findings only hint at the long-term potential of economic analyses in prevention research. That potential will be reached when researchers and policy makers can compare costs and effectiveness across disparate programs to prevent mental disorder among funds as a portfolio which they can optimize (Sendi et al. 2004; Simons 2003; Sendi et al. 2003; Bridges et al. 2002). Policy makers would use economic analyses not only to pick among competing interventions targeted to the same disorder but would use that information to set research and services funding across disorders. 
Regrettably, we are a long way from that point. A variety of problems prevent us from comparing cost-effectiveness across interventions. A necessary first step is to ensure that the estimates of program costs are comparable across studies. As our discussion above reveals, current studies are difficult or impossible to compare because of differences in methodology. The problems with prior studies is not that those studies are wrong, but as our example makes clear, any attempt to estimate costs involves choices and assumptions. We encourage authors to report their cost estimates in enough detail that those assumptions are apparent and, wherever possible, report sensitivity analyses that indicate whether and how their estimates vary across reasonable alternatives. Then when other researchers make other choices, those differences will not prevent one from comparing cost findings across studies.

Finally, our article is fairly unique in that we take a first, small step toward comparing costs for interventions when delivered as part of efficacy studies and by real-world providers. We know of no studies that have attempted to do so formally. As more interventions move from efficacy to effectiveness studies, whether and how the real-world costs vary is an important area for future research. 
Table 1

Intervention costs from a public agency perspective (explicit costs)

\begin{tabular}{lrrr}
\hline Explicit Costs & Per Family $(N=90)$ & Per Person $(N=243)$ & Per Hour of Contact \\
\hline Cohort 1 $(n=39)$ & $\$ 4,487$ & $\$ 1,611$ & $\$ 59$ \\
Cohort 2 $(n=42)$ & $\$ 4,228$ & $\$ 1,611$ & $\$ 59$ \\
Cohort 3 $(n=37)$ & $\$ 3,766$ & $\$ 1,629$ & $\$ 59$ \\
Cohort 4 $(n=32)$ & $\$ 6,957$ & $\$ 2,174$ & $\$ 79$ \\
Cohort 5 $(n=32)$ & $\$ 4,375$ & $\$ 1,777$ & $\$ 65$ \\
Cohort 6 $(n=61)$ & $\$ 2,731$ & $\$ 940$ & $\$ 34$ \\
Mean & $\$ 4,424$ & $\$ 1,624$ & $\$ 59$ \\
\hline
\end{tabular}


Table 2

Intervention costs from a societal perspective (explicit and implicit costs)

\begin{tabular}{|c|c|c|c|}
\hline Explicit \& Implicit & Per Family $(N=90)$ & Per Person $(N=243)$ & Per Hour of Contact \\
\hline Cohort $1(n=39)$ & $\$ 5,004$ & $\$ 1,796$ & $\$ 65$ \\
\hline Cohort $2(n=42)$ & $\$ 4,726$ & $\$ 1,800$ & $\$ 65$ \\
\hline Cohort $3(n=37)$ & $\$ 4,233$ & $\$ 1,830$ & $\$ 67$ \\
\hline Cohort $4(n=32)$ & $\$ 7,799$ & $\$ 2,437$ & $\$ 89$ \\
\hline Cohort $5(n=32)$ & $\$ 4,918$ & $\$ 1,998$ & $\$ 73$ \\
\hline Cohort $6(n=61)$ & $\$ 3,354$ & $\$ 1,155$ & $\$ 42$ \\
\hline Mean & $\$ 5,006$ & $\$ 1,836$ & $\$ 67$ \\
\hline
\end{tabular}


Table 3

Breakdown of explicit costs

\begin{tabular}{|c|c|c|c|c|c|c|c|}
\hline Explicit Costs & Cohort 1 & Cohort 2 & Cohort 3 & Cohort 4 & Cohort 5 & Cohort 6 & Mean \\
\hline \multicolumn{8}{|c|}{ Resources directly related to service delivery } \\
\hline $\begin{array}{l}\text { Training, } \\
\text { Supervision, } \\
\text { Faculty Time }\end{array}$ & $\$ 13,636$ & $\$ 13,890$ & $\$ 12,337$ & $\$ 17,601$ & $\$ 13,189$ & $\$ 14,534$ & $\$ 14,198$ \\
\hline $\begin{array}{l}\text { Clinical } \\
\text { Support }\end{array}$ & $\$ 0$ & $\$ 4,200$ & $\$ 5,500$ & $\$ 4,900$ & $\$ 2,600$ & $\$$ & $\$ 2,867$ \\
\hline Group Leader & $\$ 27,149$ & $\$ 33,768$ & $\$ 26,492$ & $\$ 29,778$ & $\$ 25,858$ & $\$ 27,496$ & $\$ 28,424$ \\
\hline Sub-total & $\$ 40,785$ & $\$ 51,858$ & $\$ 44,329$ & $\$ 52,279$ & $\$ 41,647$ & $\$ 42,030$ & \\
\hline \multicolumn{8}{|c|}{ Intervention Support } \\
\hline Hourly Staff & $\$ 3,271$ & $\$ 3,919$ & $\$ 3,101$ & $\$ 3,300$ & $\$ 1,964$ & $\$ 1,376$ & $\$ 2,822$ \\
\hline Administrative & $\$ 3,152$ & $\$ 922$ & $\$ 810$ & $\$ 983$ & $\$ 910$ & $\$ 994$ & $\$ 1,295$ \\
\hline Consultants & $\$ 2,114$ & $\$ 50$ & $\$ \quad 0$ & $\$ 391$ & $\$ 215$ & $\$ 527$ & $\$ 550$ \\
\hline Benefits & $\$ 3,022$ & $\$ 2,666$ & $\$ 2,460$ & $\$ 3,345$ & $\$ 2,538$ & $\$ 2,795$ & $\$ 2,804$ \\
\hline Services & $\$ 8,868$ & $\$ 5,214$ & $\$ 7,760$ & $\$ 8,183$ & $\$ 8,039$ & $\$ 7,590$ & $\$ 7,609$ \\
\hline Supplies & $\$ 797$ & $\$ 2,222$ & $\$ 1,000$ & $\$ 285$ & $\$ 757$ & $\$ 1,242$ & $\$ 1,051$ \\
\hline Equipment & $\$ 804$ & $\$ 804$ & $\$ 804$ & $\$ 804$ & $\$ 804$ & $\$ 804$ & $\$ 804$ \\
\hline Total & $\$ 62,813$ & $\$ 67,655$ & $\$ 60,264$ & $\$ 69,470$ & $\$ 56,874$ & $\$ 57,358$ & \\
\hline
\end{tabular}


Table 4

Development and pilot intervention costs (explicit and implicit costs)

\begin{tabular}{lllll}
\hline & \multicolumn{2}{c}{ Pilot I $(n=14)$} & \multicolumn{2}{c}{ Pilot II $(n=16)$} \\
\hline Explicit & Total Costs & Per Family & Total Costs & Per Family \\
Intervention & $\$ 16,403$ & $\$ 1,172$ & $\$ 16,403$ & $\$ 1,025$ \\
Development & $\$ 45,601$ & $\$ 3,257$ & $\$ 1,412$ & $\$ 88$ \\
Explicit and Implicit & & & & \\
\hline Intervention & $\$ 18,671$ & $\$ 1,334$ & $\$ 18,671$ & $\$ 1,167$ \\
Development & $\$ 78,051$ & $\$ 5,575$ & $\$ 1,208$ & $\$ 76$ \\
\hline
\end{tabular}


Table 5

Proportion of Group Facilitator time spent in various intervention related activities during efficacy trial and within a natural service delivery setting (real world).

\begin{tabular}{lcc}
\hline Activity & Efficacy & Real World \\
\hline Group Intervention & $28 \%$ & $50 \%$ \\
Training & $24 \%$ & $7 \%$ \\
Supervision & $7 \%$ & $2 \%$ \\
Co-Leader Practice & $2 \%$ & $0 \%$ \\
Preparation for intervention & $20 \%$ & $9 \%$ \\
Clinical notes & $12 \%$ & $20 \%$ \\
Interviews & $4 \%$ & $7 \%$ \\
Telephone calls & $2 \%$ & $4 \%$ \\
& $100 \%$ & $100 \%$ \\
\hline
\end{tabular}




\section{References}

Aos, Steve, Roxanne Lieb, Jim Mayfield, Marna Miller, and Annie Pennucci. 2004. Benefits and costs of prevention and early intervention programs for youth. Seattle, WA: Washington State Public Policy Institute.

Barnett, W. Steven. 1996. Lives in the Balance: Age-27 Benefit-Cost Analysis of the High/Scope Perry Preschool Program. Ypsilanti, Michigan: High/Scope.

Bickman, Leonard, Pamela R. Guthrie, E. Michael Foster, E. Warren Lambert, Thomas Wm. Summerfelt, Carolyn Breda, and Craig Anne Heflinger. 1995. Evaluating managed mental health services: The Fort Bragg experiment. New York: Plenum Press.

Borjas, George J. 2005. Labor economics. 3rd ed. Boston: McGraw-Hill/Irwin.

Bridges, J. F., M. Stewart, M. T. King, and K. van Gool. 2002. Adapting portfolio theory for the evaluation of multiple investments in health with a multiplicative extension for treatment synergies. Eur J Health Econ 3 (1):47-53.

Browning, Edgar K., and Jacquelene M. Browning. 1992. Microeconomic Theory and Applications. 4th edition ed. New York: HarperCollins Publishers Inc.

Ehrenberg, Ronald G., and Robert Stewart Smith. 2006. Modern labor economics : theory and public policy. 9th ed, The Addison-Wesley series in economics. Boston: Pearson/Addison Wesley.

Gold, Martha R., Louise B. Russell, Joanna E. Siegel, and Milton C. Weinstein, eds. 1996. Costeffectiveness in health and medicine. New York: Oxford University Press.

Gregory, I. 1965. Anterospective data following childhood loss of a parent. Archives of General Psychiatry 13:99-109.

Haddix, Anne C., Steven M. Teutsch, and Phaedra S. Corso, eds. 2003. Prevention effectiveness: 
A Guide to decision analysis and economic evaluation. Second ed. Oxford: Oxford University Press.

Hargreaves, William A., Martha Shumway, T. W. Hu, and Brian Cuffel. 1998. Cost-outcome methods for mental health. New York: Academic Press.

Karoly, Lynn A., M. Rebecca Kilburn, James H. Bigelow, Johathan P. Caulkins, and Jill S. Cannon. 2001. Assessing Costs and Benefits of Early Childhood Intervention Programs: Overview and Application to the Starting Early Starting Smart Program. Santa Monica, CA: Rand.

Maase, Leonard N., and W. Steven Barnett. 2003. A Benefit cost analysis of the Abecedarian early childhood intervention. New Brunswick, NJ: National Institute for Early Education Research.

Olds, David L., Charles R. Henderson, Charles Phelps, Harriet Kitzman, and Carole Hanks. 1993. Effect of Prenatal and Infancy Nurse Home Visitation on Government Spending. Medical Care 31 (2):155-174.

Posnett, J., and S. Jan. 1996. Indirect cost in economic evaluation: the opportunity cost of unpaid inputs. Health Economics 5:13-23.

Reynolds, Arthur J., Judy A. Temple, Dylan L. Robertson, and Emily A. Mann. 2002. Age 21 Cost-Benefit Analysis of the Title 1 Chicago Child-Parent Centers. Madison, WI: Institute for Research on Poverty.

Sendi, P., M. J. Al, A. Gafni, and S. Birch. 2003. Optimizing a portfolio of health care programs in the presence of uncertainty and constrained resources. Soc Sci Med 57 (11):2207-15.

— 2004. Portfolio theory and the alternative decision rule of cost-effectiveness analysis: theoretical and practical considerations. Soc Sci Med 58 (10):1853-5. 
Simons, J. 2003. The perfect mix for your portfolio. Fortune 148 (13):134-8.

Spoth, Richard L., Max Guyll, and Susan X. Day. 2002. Universal family-focused interventions in alcohol-use disorder prevention: cost-effectiveness and cost-benefit analyses of two interventions. Journal of Studies on Alcohol 63:219-228.

Thompson, Mark S. 1980. Benefit-cost analysis for program evaluation. Beverly Hills, Calif.: Sage Publications. 